\title{
Desert Hedgehog Links Transcription Factor Sox10 to Perineurial Development
}

\author{
Melanie Küspert, ${ }^{1}$ Matthias Weider, ${ }^{1}$ Jana Müller, ${ }^{1}$ Irm Hermans-Borgmeyer, ${ }^{2}$ Dies Meijer, ${ }^{3}$ and Michael Wegner ${ }^{1}$ \\ ${ }^{1}$ Institute of Biochemistry, Emil-Fischer-Zentrum, Friedrich-Alexander University Erlangen-Nürnberg, D-91054 Erlangen, Germany, ${ }^{2}$ Center for Molecular \\ Biology Hamburg, University Medical Center Eppendorf, D-20251 Hamburg, Germany, and 32Department of Cell Biology and Genetics, Erasmus MC, 3015 \\ GE Rotterdam, The Netherlands
}

Schwann cells are the main glial cell type in the PNS. They develop along nerves during embryogenesis and rely on the HMG domain containing Sox 10 transcription factor for specification, lineage progression, and terminal differentiation. Sox10 deletion in immature Schwann cells caused peripheral nerve defects in mice that were not restricted to this glial cell type, although expression in the nerve and gene loss were. Formation of the perineurium as the protecting sheath was, for instance, heavily compromised. This resembled the defect observed after loss of Desert hedgehog $(D h h)$ in mice. Here we show that Sox 10 activates Dhh expression in Schwann cells via an enhancer that is located in intron 1 of the Dhh gene. Sox 10 binds this enhancer in monomeric form via several sites. Mutation of these sites abolishes both Schwann-cell-specific activity and Sox10 responsiveness in vitro and in transgenic mouse embryos. This argues that Sox 10 activates Dhh expression by direct binding to the enhancer and by increasing Dhh levels promotes formation of the perineurial sheath. This represents the first mechanism for a non-cell-autonomous function of Sox 10 during peripheral nerve development.

\section{Introduction}

Sox10 regulates several aspects of vertebrate nervous system development (Stolt and Wegner, 2010). In the CNS, it is selectively expressed in oligodendrocyte lineage cells in which it affects several stages of development and is indispensable for terminal differentiation and myelination (Stolt et al., 2002; Finzsch et al., 2008). In the forming PNS, Sox10 influences the generation of several cell types, including sympathetic and sensory neurons (Kim et al., 2003; Carney et al., 2006; Reiprich et al., 2008). Even more important is its requirement for the specification of all glia, including Schwann cell precursors along nerves (Britsch et al., 2001; Paratore et al., 2001).

In Schwann cells, Sox10 is expressed throughout development (Kuhlbrodt et al., 1998a). After its role in specification (Britsch et al., 2001), it continues to be functional as Schwann cell precursors develop first into immature Schwann cells and then via promyelinating to myelinating Schwann cells. This lasting requirement has been confirmed by the analyses of mice with hypomorphic Sox10 alleles and mice in which a loxP-flanked Sox10 allele was deleted during specific stages of Schwann cell development by Cre recombinase (Schreiner et al., 2007; Finzsch et al., 2010; Bremer et al., 2011; Fröb

Received Nov. 18, 2011; revised Feb. 28, 2012; accepted Feb. 29, 2012.

Author contributions: M.K. and M.Weg. designed research; M.K., M.Wei., J.M., and I.H.-B. performed research; D.M. contributed unpublished reagents/analytic tools; M.K., M.Wei., I.H.-B., and M.Weg. analyzed data; M.K. and M.Weg. wrote the paper.

This work was supported by Deutsche Forschungsgemeinschaft Grant We1326/9 (M.Weg.). Sarah Homann is acknowledged for help with transgenesis.

Correspondence should be addressed to Dr. Michael Wegner, Institut für Biochemie, Emil-FischerZentrum, Friedrich-Alexander Universität, Fahrstrasse 17, 91054 Erlangen, Germany. E-mail: m.wegner@ biochem.uni-erlangen.de.

DOI:10.1523/JNEUROSCI.5759-11.2012

Copyright $\odot 2012$ the authors $\quad 0270-6474 / 12 / 325472-09 \$ 15.00 / 0$ et al., 2012). Although the essential role of Sox10 for Schwann cell development is evident, much remains to be learned about its mode of action. One of the key issues is the identification of stage-specific Sox10 target genes that are themselves important for peripheral nerve development.

Sox10 activates the expression of peripheral myelin genes during terminal differentiation, including $M a g, M b p, M p z, P m p 22$, and Connexin 32 (Peirano et al., 2000; Bondurand et al., 2001; Jones et al., 2007, 2011). Sox10 is also directly involved in the activation of Krox20, which is a key activator of peripheral myelin genes (Ghislain and Charnay, 2006; Reiprich et al., 2010). Available evidence suggests that Sox10 first activates and then cooperates with Krox20 to activate the myelination program (Svaren and Meijer, 2008).

Two other Sox10 targets are the genes for the POU domain transcription factor Oct6 and the receptor tyrosine kinase subunit ErbB3 (Jagalur et al., 2011; Prasad et al., 2011). Oct6 is required for Schwann cells to progress to the pro-myelinating stage and to prepare for terminal differentiation (Bermingham et al., 1996; Jaegle et al., 1996), whereas ErbB3 acts as part of the ErbB2/ ErbB3 heterodimer that allows Schwann cells to respond in various phases of their development to neuregulin 1 (Newbern and Birchmeier, 2010). Although these known targets help to explain several Sox10 functions, they are by no means sufficient to fully understand the mechanisms by which Sox10 influences peripheral nerve development.

We have discovered recently that Sox10 deletion in immature Schwann cells also led to a dramatic decrease in Dhh levels (Finzsch et al., 2010). Here we show that Dhh is also a direct target gene of Sox10 in Schwann cells and that, through its influence on $D h h$, Sox10 exerts a non-cell-autonomous effect on perineurial cells and the formation of the perineurium. 


\section{Materials and Methods}

Plasmids. The Dhh genomic region analyzed in this study was located between positions 98720506 and 98739191 of mouse chromosome 15 (mouse genome version mm9). From this region, fragments were obtained by PCR or using restriction nucleases, as indicated in Figures $1 A$, $2 A$, and $3 A$, and inserted into reporter plasmids. For autofluorescencebased reporter gene studies, $p T A T A-t d T o m a t o$ was used. It carried the red fluorescing GFP derivative $t d$ Tomato under control of the $\beta$-globin minimal promoter. For luminescence-based reporter gene assays, Dhhluc was used. This plasmid is based on pGL2basic (Promega) and contained the firefly luciferase gene downstream of the Dhh minimal promoter (positions -277 to +31 relative to the transcriptional start site). In the context of the E4-Dhh-luc construct (see Fig. $3 A$ ), potential Sox10 binding sites were mutated using the QuickChange XL SiteDirected Mutagenesis kit (Stratagene). The eukaryotic $p C M V 5$-based expression plasmids for full-length Sox10 ( $p$ CMV5-Sox10) and the short MIC version of Sox10 ( $p$ CMV5-Sox10 MIC) have been described as well as the $p$ CAGGS-Sox10-IRES-nls-GFP plasmid in which Sox10 and GFP reading frames are under control of the chicken $\beta$-actin promoter (Kuhlbrodt et al., 1998b; Cossais et al., 2010). For knockdown experiments, pSuper-Neo-GFP plasmids (Oligoengine) were used that either expressed a Sox10-specific shRNA (targeted region, 5'-CTGCTGTTCC TTCTTGACCT TGCCC-3') or a control shRNA. E4-lacZ transgene constructs contained the $E 4$ fragment in wild-type or mutant version (see Fig. $7 C$ ) from the Dhh locus in front of the Dhh minimal promoter followed by a lacZ reporter gene and poly(A) cassette.

Cell culture, transient transfection, extract preparation, electrophoretic mobility shift assays, and reporter gene assays. HEK293 cells were maintained in DMEM containing 10\% fetal calf serum (FCS) and transfected by the polyethylenimine technique using $10 \mu \mathrm{g}$ of pCMV5-based expression plasmid per $100 \mathrm{~mm}$ plate. At $48 \mathrm{~h}$ after transfection, cells were harvested for extract preparation (Schlierf et al., 2002). After verification of ectopic expression by Western blotting, electrophoretic mobility shift analyses (EMSAs) were performed (Küspert et al., 2011) using ${ }^{32} \mathrm{P}$ labeled 25 bp double-stranded oligonucleotides containing putative Sox10 binding sites.

For reporter gene assays, rat S16 Schwann cells were maintained in DMEM containing 10\% FCS and transfected with SuperFect Transfection Reagent (Qiagen) on 24-well tissue culture plates. For autofluorescence-based assays, cells were transfected with $500 \mathrm{ng}$ of pTATA-tdTomato-based reporter plasmids and scored for fluorescence $48 \mathrm{~h}$ after transfection using a Leica inverted microscope (DMIRB) equipped with a cooled MicroMax CCD camera (Princeton Instruments). For luminescence-based assays, $500 \mathrm{ng}$ of Dhh-lucbased reporter plasmids were used in the presence of $100 \mathrm{ng}$ of pCMV5- or pSuper-Neo-GFP-based expression vectors. Cells were generally harvested $48 \mathrm{~h}$ after transfection, except for knockdown experiments in which analysis took place $72 \mathrm{~h}$ after transfection. Luciferase activity was determined in the presence of luciferin substrate by detection of chemiluminescence.

Chromatin immunoprecipitation. Chromatin immunoprecipitation (ChIP) assays were performed on S16 cells as described previously (Schlierf et al., 2006). After crosslinking proteins to DNA in the presence of $1 \%$ formaldehyde, chromatin was prepared and sheared to an average fragment length of 200-500 bp using a Sonoplus HD2070 homogenizer (Bandelin). Immunoprecipitations were performed overnight at $4^{\circ} \mathrm{C}$ using guinea pig antiserum against Sox10 (1:400 dilution) (Maka et al., 2005) as well as guinea pig preimmune serum and protein A Sepharose beads. Quantitative PCR was performed on input and precipitated chromatin after crosslink reversal and purification. The following primer pairs were used at an annealing temperature of $63^{\circ} \mathrm{C}: 5^{\prime}$-GCAGCCAAGA TAACTGTGGC- $3^{\prime}$ and $5^{\prime}$-GCAGTACAATGGCCATTCTC-3' for E4a; $5^{\prime}$-CAATGCCCAGTGCCAGGGAAG-3' and $5^{\prime}$-CTCCCAGCGTTTGG GAGTCG-3' for $E 4 b ; 5^{\prime}$-GGCAGAGAGCTGGGATTGTC-3' and $5^{\prime}$-CC AGGGTTGGCCTATACACG-3' for Con1; and 5'-CAATTGACATAT GCCAGCCC-3' and 5'-GATCACACATCTAAGGCCTC-3' for Con2.

Generation and analysis of transgenic animals. Transgenic mouse embryos were generated by microinjecting the E4-lacZ transgene in wild- type or mutant form (E4m3-lacZ and E4mAll-lacZ; see Fig. 7C) as an NheI/KpnI fragment into the male pronucleus of fertilized F1 (C57BL/ $6 \times$ CBA) oocytes according to standard techniques. Foster mothers were killed when embryos were at $14.5 \mathrm{~d}$ postcoitum. Transgenic embryos were identified and genotyped by PCR on embryonic tail DNA using 5' -GCCCAGGAAGATAGTTTGGTG-3' and 5' -GATAGGTTAC GTTG GTGTAGATGG-3' as primers under standard PCR conditions.

The E4-lacZ transgene was injected as plasmid into the neural tube of live chicken embryos at Hamburger-Hamilton stage 10-11 in the presence of $p C A G G S-I R E S-n l s-G F P$-based expression plasmids and electroporated as described previously (Cossais et al., 2010). Successfully electroporated embryos were identified $48 \mathrm{~h}$ later by GFP expression and dissected.

After fixation, mouse and chicken embryos were cryoprotected in sucrose and frozen at $-80^{\circ} \mathrm{C}$ in tissue freezing medium (Leica). After transverse sectioning on a cryotome, $\beta$-galactosidase activity was detected on $20 \mu \mathrm{m}$ sections by incubation in $1 \% \mathrm{X}$-gal at $37^{\circ} \mathrm{C}$. For immunohistochemistry, 10 $\mu \mathrm{m}$ cryotome sections were incubated with anti- $\beta$-galactosidase goat antiserum (1:500 dilution; Biotrend), anti-Sox10 guinea pig antiserum (1:1000 dilution) (Maka et al., 2005), or anti-GFP mouse monoclonal antibody (1: 100 dilution; Roche). Secondary antibodies conjugated to Cy2, Alexa Fluor 488, and Cy3 immunofluorescent dyes (Dianova and Molecular Probes) were used for detection. Sections were analyzed and documented with either a Leica TCS SL confocal microscope or a Leica MZFLIII stereomicroscope equipped with an Axiocam (Carl Zeiss).

\section{Results}

\section{The first intron of the Dhh gene has enhancer activity in S16} Schwann cells

Previous studies had shown that Dhh is not only strongly expressed in the male gonad but also in the developing peripheral nerve in which it is predominantly produced from Schwann cells (Bitgood and McMahon, 1995; Parmantier et al., 1999). Loss of this paracrine signaling molecule in mice leads to massive defects of the perineurium, the cellular sheath that surrounds and protects the endoneurium with its axon-Schwann cell units and blood vessels (Parmantier et al., 1999). It also prevents invasion of immune cells into the nerve. The perineurium consists of several layers of flattened fibroblasts that secret collagens and other extracellular matrix molecules as well as poorly characterized signaling factors. Many of the extracellular matrix molecules end up in the epineurium and thereby help to form the outer nerve cover. The signaling molecules from perineurial cells influence many aspects of nerve development. In the absence of Dhh, the perineurium no longer provides an effective barrier between surrounding tissue and nerve. It appears thin, less compacted, and disorganized, with patchy basal lamina and abnormal tight junctions (Parmantier et al., 1999). In Dhh-deficient mice, perineurial cells divide the nerve into mini-fascicles. Alterations in perineurium-derived signals cause defects in myelinating as well as non-myelinating Schwann cells. They lead to an abnormal permeability of the blood-nerve barrier and elevated levels of immune cells in the nerve (Sharghi-Namini et al., 2006). Similar consequences have also been observed in humans in which Dhh loss leads to a peripheral neuropathy with mini-fascicle formation and gonadal dysgenesis (Umehara et al., 2000).

Here we analyzed whether Dhh is a direct target of Sox 10 . Transcription of the Dhh gene has not been studied, and the regulatory region that mediates its expression in Schwann cells has not yet been identified. However, work by Jaegle et al. (2003) had shown that $19 \mathrm{~kb}$ of genomic sequence around the Dhh gene are sufficient to achieve expression of a Cre transgene in Schwann cells. This region encompasses all three Dhh exons as well as upstream and downstream sequences. To identify the part responsible for Schwann-cell-specific expression, we divided the 
Dhh locus in eight overlapping fragments (Fig. 1A) and combined each with the $\beta$-globin minimal promoter (Fig. $1 B$ ). All eight combinations were then tested for their ability to drive expression of a tdTomato reporter gene in the S16 Schwann cell line using autofluorescence of transfected cells as readout (Fig. 1C). Combinations of the same $\beta$-globin minimal promoter with the U3 enhancer of the Sox 10 gene or with the enhancer of the $M p z$ gene served as controls and verified that the assay is sensitive enough to detect the activity of Schwann-cell-specific enhancers (LeBlanc et al., 2007; Werner et al., 2007).

In contrast to the two known Schwann cell enhancers, none of the four fragments from the upstream region of the Dhh gene (F1-F4; Fig. 1A) exhibited autofluorescence (Fig. 1C). Equally inactive as the upstream fragments was F5. This fragment spanned the immediate upstream region, exon 1 , and part of intron 1 and contained the Dhh promoter. It follows that the promoter is not sufficient to secure more than basal expression in S16 cells. In contrast, autofluorescence was observed with fragment F6 (Fig. 1A,C). This contained all three exons of the Dhh gene, the intervening introns, and the immediate downstream region. A comparable autofluorescence was also obtained with a fragment that corresponded to intron 1 (F7; Fig. $1 A, C$ ), whereas a fragment representing the complete downstream region remained inactive (F8; Fig. $1 A, C$ ). These results point to enhancer activity in intron 1.

Autofluorescence was fairly weak and is inconvenient for quantification. We were furthermore concerned that enhancer activity may be suboptimal in combination with the heterologous $\beta$-globin promoter. Therefore, we switched to a luciferase reporter assay and exchanged the $\beta$-globin promoter for a 308-bp-long Dhh minimal promoter $(-277$ to +31$)$ (Fig. $2 B$ ). This minimal promoter increased luciferase activity barely above levels observed with the promoterless construct. High activity was observed in S16 cells not before the Dhh minimal promoter was combined with the $U 3$ enhancer. This construct served again as positive control (Fig. 2C). In contrast, combination of the Dhh minimal promoter with the Sox10 D7 enhancer remained inactive, in agreement with its lack of activity in Schwann cells (Werner et al., 2007).

As observed for combinations with the $\beta$-globin promoter, none of the fragments from the Dhh upstream region (F9-F12; Fig. 2A) nor any from the downstream region (F15 and F16; Fig. $2 A$ ) showed activity in $\mathrm{S} 16$ cells when combined with the Dhh promoter (Fig. 2C). Activity was instead confined to F13 and F14. These fragments strongly overlapped and contained

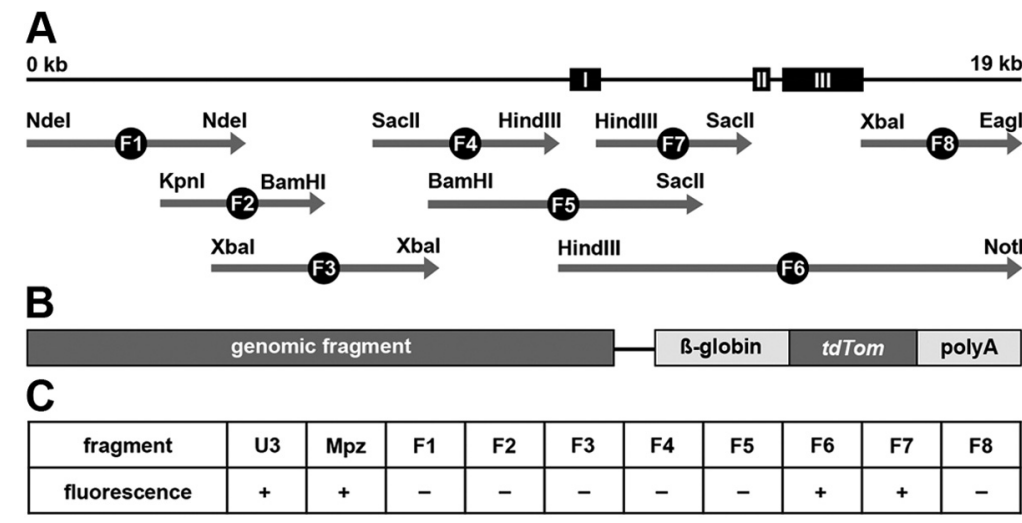

Figure 1. Scanning the Dhh genomic region for an enhancer. $A$, Topology of the Dhh genomic region and localization of the fragments $F 1-F 8$ in this $19 \mathrm{~kb}$. Restriction enzymes used for generation of the fragments are indicated. $\boldsymbol{B}$, Scheme of the reporter construct. Fragments $F 1-F 8$ were inserted in front of the $\beta$-globin minimal promoter, followed by $t d T o m a t o$ and SV40 poly(A) signal. C, At $48 \mathrm{~h}$ after transient transfection of the reporter plasmids, S16 cells were scored for expression of the reporter by detection of tdTomato-specific autofluorescence. The experiment was repeated three times in duplicates. Reporter plasmids that carried the U3 enhancer or the Mpz enhancer in combination with the $\beta$-globin minimal promoter served as positive control. + , Autofluorescence detected; - , no autofluorescence.

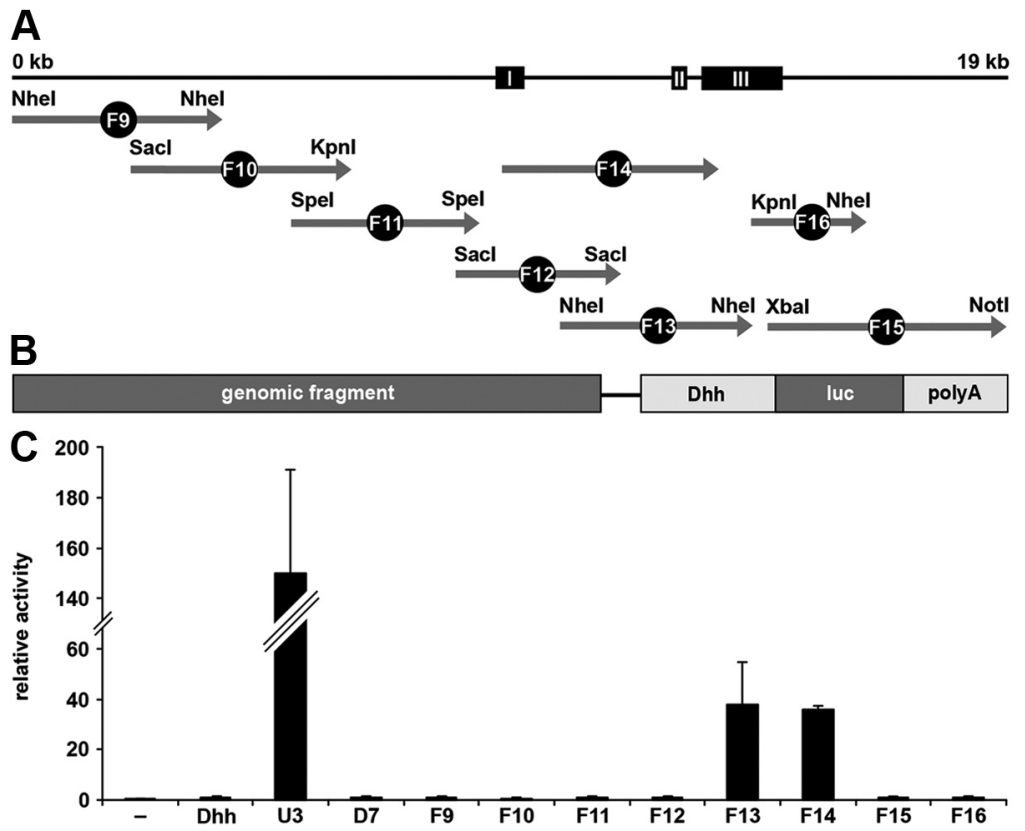

Figure 2. Mapping enhancer activity to intron 1 of the Dhh gene. $A$, Schematic representation of fragments $F 9-F 16$ and their location relative to the Dhh gene. Restriction sites used for cloning are indicated at the ends of each fragment. F14 was generated by PCR. $\boldsymbol{B}$, Reporter constructs consisted of fragments F9-F16 inserted in front of Dhh minimal promoter (positions -277 to +31 , Dhh), luciferase gene, and SV40 poly(A) signal. C, Transient transfections of these reporter constructs were performed in S16 cells. Luciferase activities in extracts from transfected cells were determined $48 \mathrm{~h}$ after transfection in three experiments each performed in duplicates. The luciferase activity obtained for a reporter plasmid containing only the minimal Dhh promoter (Dhh) was arbitrarily set to 1 . Activities in the presence of additional Dhh regions were calculated relative to minimal promoter activity and are presented as mean $\pm S D$. A reporter in which the Dhh minimal promoter was combined with the $U 3$ enhancer served as positive control, one in which it was combined with the D7 enhancer as negative control. - , Reporter plasmid without the minimal promoter.

most of the sequences from intron 1, confirming that this intron likely contains the enhancer element.

\section{The Dhh enhancer is confined to the second half of intron 1}

To determine more exactly the location of the enhancer, we generated subfragments of F14 (Fig. 3A) and tested them again in S16 cells for their ability to induce expression of a luciferase reporter (Fig. 3B). Division of F14 into a larger $2.8 \mathrm{~kb}$ fragment spanning the first two-thirds and a smaller $1.4 \mathrm{~kb}$ one containing the remaining one-third led to the two, equally inactive F17 and F18 fragments. 

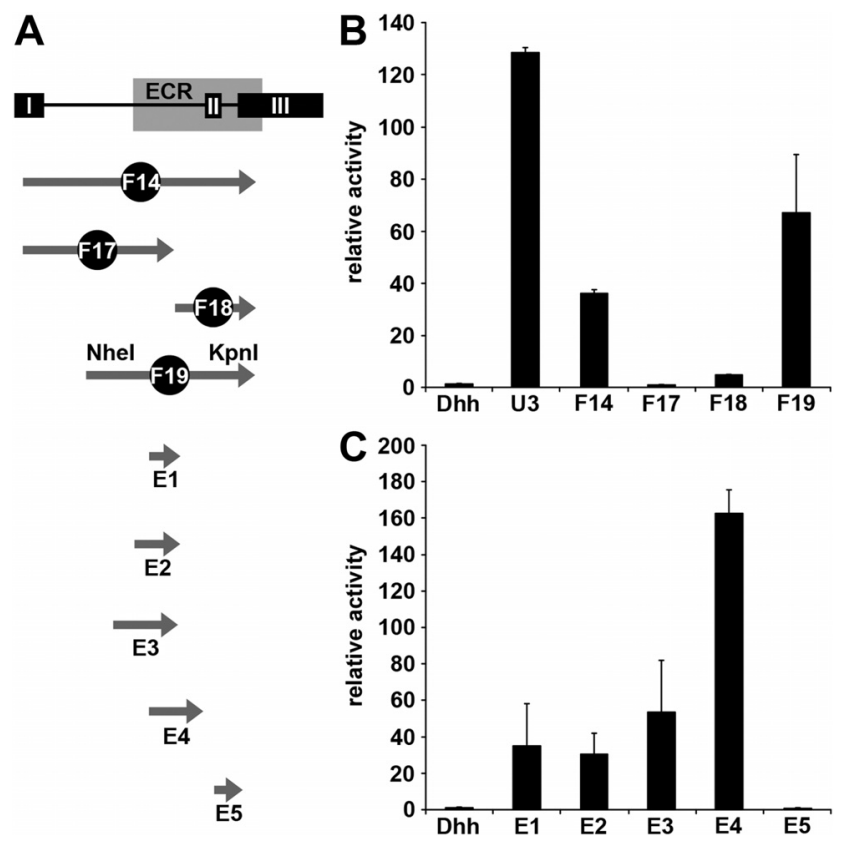

Figure 3. Fine mapping of the Dhh enhancer to an evolutionary conserved region in the hind part of intron 1.A, Location of fragments $F 14, F 17-F 19$, and E1-E5 relative to exons I-III of the Dhh gene and the region with highest evolutionary conservation $(E C R)$ in the gene. Restriction sites used for cloning are indicated. All other fragments were amplified by PCR. B, C, Luciferase reporter constructs containing one of the $F(\boldsymbol{B})$ or $E(\boldsymbol{C})$ fragments inserted in front of the Dhh minimal promoter were transiently transfected in $\mathrm{S} 16$ cells, and luciferase activities were determined $48 \mathrm{~h}$ after transfection in three experiments each performed in duplicate. The luciferase activity obtained for a reporter plasmid containing only the minimal Dhh promoter (Dhh) was arbitrarily set to 1 . Activities in the presence of additional Dhh regions were calculated relative to minimal promoter activity and are presented as mean \pm SD.

In contrast, enhancer activity was preserved in a $2.8 \mathrm{~kb}$ fragment that corresponded to the hind two-thirds of F14 (Fig. 3B).

This F19 fragment furthermore strongly overlapped with a region that exhibits conservation among mammals and reaches from the middle of intron 1 into exon 3 (Fig. $3 A$, ECR). Conservation is, however, confined to mammals and not seen in other vertebrates. Restricting our search to the evolutionary conserved part of F19, we generated five more fragments (Fig. 3A, E1-E5) and combined them with the Dhh minimal promoter in reporter plasmids to assess their enhancer activity in S16 cells. Of these five fragments, all except E5 exhibited enhancer activity (Fig. 3C). Activity varied considerably, with highest levels being found in E4. Therefore, we regard the 927-bp-long E4 region in intron 1 as the core of the Dhh enhancer.

\section{The Dhh enhancer is responsive to Sox10}

Because Dhh expression may be under control of Sox10 in Schwann cells (Finzsch et al., 2010), we wanted to find out whether activity of the newly identified Dhh enhancer depends on Sox10. To interfere with the function of endogenous Sox10 in S16 cells (Reiprich et al., 2010) and see how this affects activity of the Dhh enhancer, we chose a dominant-negative approach. It has been shown that transfection of a Sox10 version truncated immediately behind the DNA-binding HMG domain such as Sox10 MIC (Kuhlbrodt et al., 1998b) suppresses the function of cotransfected wild-type Sox10 (Inoue et al., 2004; Prasad et al., 2011). As a consequence, reporter gene expression in S16 cells should be lowered by cotransfected Sox10 MIC for those constructs that contain Sox10-responsive sequences of the Dhh locus. A luciferase reporter containing U3 enhancer and Dhh
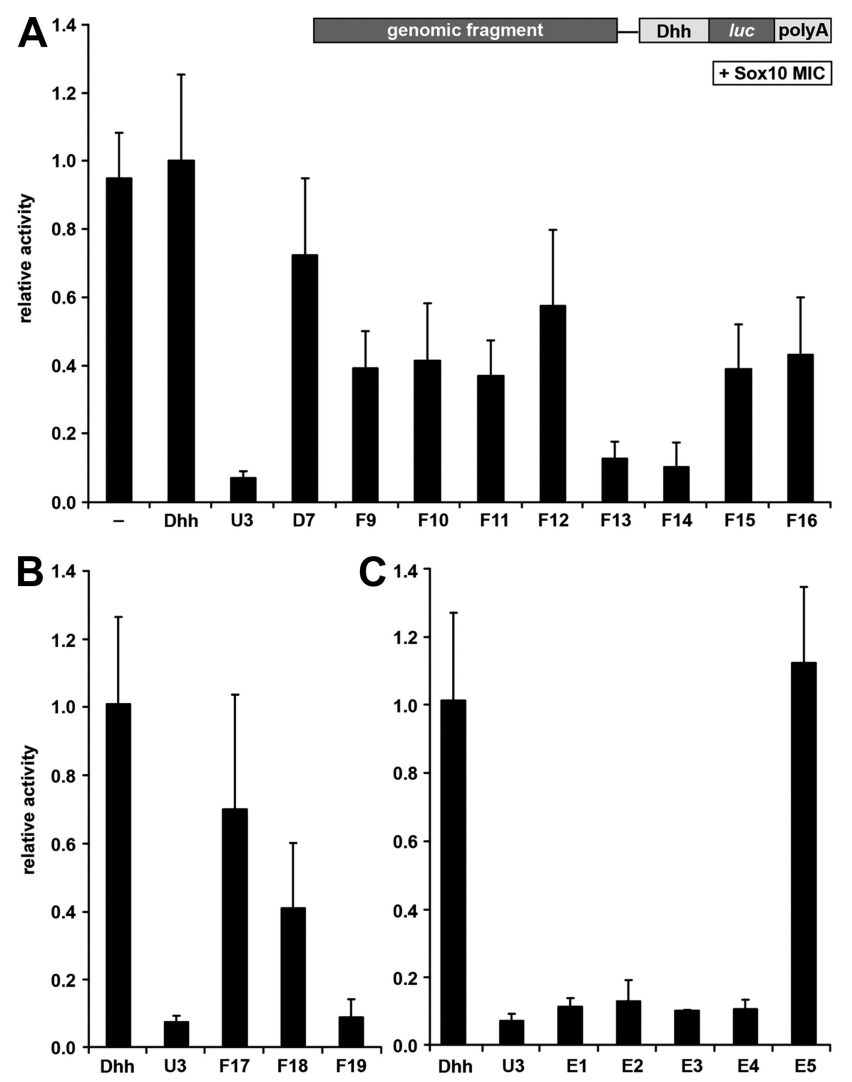

Figure 4. Sox10 responsiveness of the Dhh enhancer. Luciferase reporter constructs containing a fragment from the $D h h$ locus (see Figs. $2 A, 3 A$ ) inserted in front of $D h h$ minimal promoter and luciferase gene were transiently transfected in S16 cells in the presence of a Sox10 MIC expression plasmid or an empty expression plasmid. Luciferase activities were determined $72 \mathrm{~h}$ after transfection in three experiments each performed in duplicate. The luciferase activity obtained for a reporter plasmid in the presence of empty expression plasmid was arbitrarily set to 1. Activities in the presence of the Sox 10 MIC expression plasmid were calculated for each luciferase reporter in relation to it and are presented as mean $\pm S D$. A, Fragments $F 9-F 16$; - , reporter plasmid without the minimal promoter. $\boldsymbol{B}$, Fragments F17-F19. C, Fragments E1-E5.

minimal promoter helped to test the concept (Fig. 4A). Its activity in S16 cells was on average 16-fold lower in the presence of Sox 10 MIC. Activity of the Dhh minimal promoter alone, in contrast, was refractory to Sox10 MIC.

When the genomic Dhh fragments that were used to map the enhancer were tested for their responsiveness toward Sox10 MIC in transfected $\mathrm{S} 16$ cells by luciferase assays, many showed a slight reduction in activity. However, only those fragments that contained the enhancer were repressed more than threefold. These included fragments F13 and F14 from the original screen (Fig. $4 A$ ), as well as fragments F19 and E1-E4 from the fine mapping (Fig. $4 B, C$ ). These findings therefore support the assumption that Sox10 regulates Dhh expression in Schwann cells at least partly through this newly identified Dhh enhancer.

\section{Intron 1 of the Dhh gene contains potential binding sites} for Sox10

Activation of the Dhh enhancer may involve direct binding of Sox10. Intron 1 of the Dhh gene contains eight potential Sox binding sites, site 1 through site 8 (Fig. $5 A$ ). These were defined as sites that either conform to the Sox consensus $5^{\prime}-(\mathrm{A} / \mathrm{T})(\mathrm{A} /$ $\mathrm{T}) \mathrm{CAA}(\mathrm{A} / \mathrm{T}) \mathrm{G}-3^{\prime}$ or deviate from this consensus at no more than one position outside the central CAA core. Only site 8 carried two mismatches at the first and last positions. Each of the sites was 


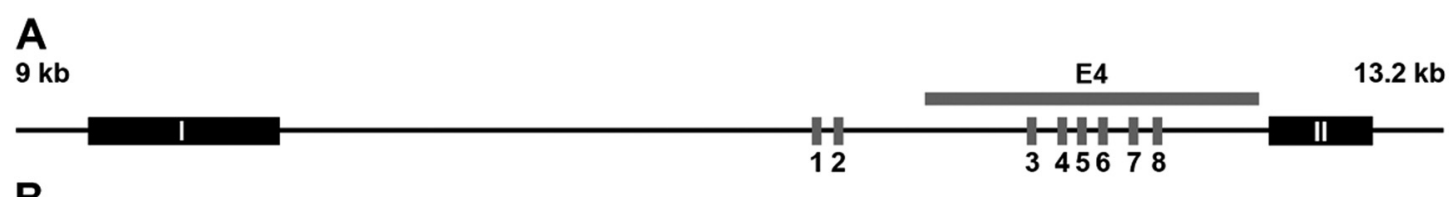

B
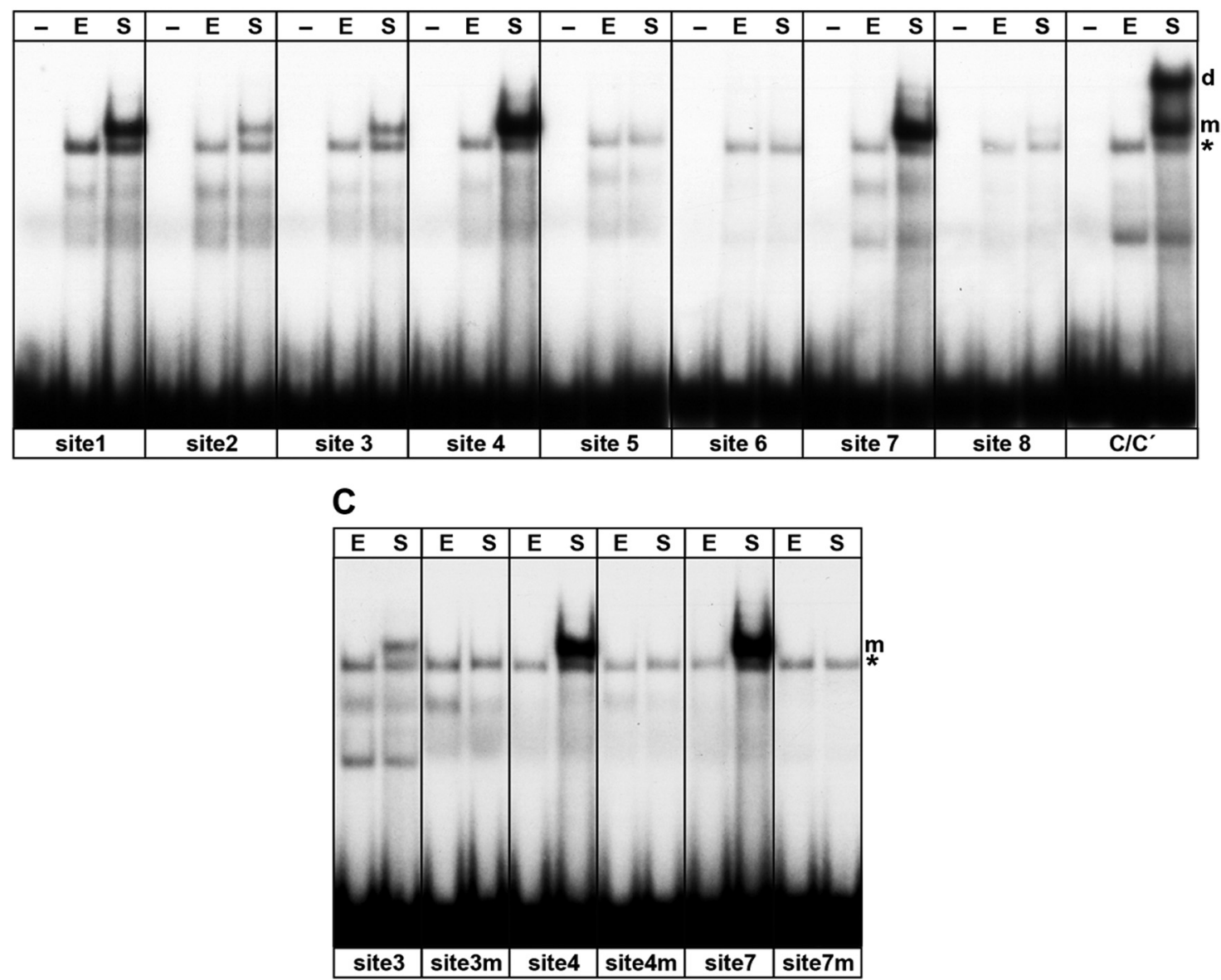

Figure 5. Mapping Sox10 binding sites in intron 1 of the Dhh gene. $\boldsymbol{A}$, Location of the eight potential Sox 10 binding sites in intron 1 and relative to the E4 fragment. $\boldsymbol{B}$, EMSA with radiolabeled double-stranded oligonucleotides encompassing one of the potential Sox10 binding sites as indicated below the gels. Oligonucleotides were incubated in the absence ( - ) or presence ( $E$, $S$ ) of protein extracts before gel electrophoresis as indicated above the lanes. Extracts were from mock-transfected HEK293 cells ( $E$ ) or HEK293 cells expressing full-length Sox 10 ( S). 0 ligonucleotides with site C/C' from the Mpz promoter (Peirano et al., 2000) served as positive control for Sox10 binding and as marker for the mobility of complexes containing either Sox10 monomers (m) or dimers (d). C, EMSA was performed with wild-type and mutant versions $(\mathrm{m})$ for binding sites 3, 4, and 7. Oligonucleotides were incubated in the presence of protein extracts from mock-transfected $\mathrm{HEK} 293$ cells $(E)$ or HEK293 cells expressing full-length Sox10 ( S). The position of the Sox10-specific complex is highlighted (m). The asterisk marks a nonspecific complex.

tested in the context of a larger oligonucleotide in EMSA with control and Sox10-containing extract. Site C/C' from the $M p z$ promoter (Peirano et al., 2000) helped to identify DNA complexes with Sox10 monomers and dimers by their respective mobility (Fig. $5 B$ ). Of the eight potential binding sites present in intron 1 , site 1 , site 4 , and site 7 bound Sox 10 strongly. Weaker binding was additionally detected to site 2 and site 3 . All of these sites bound Sox10 as monomers. Dimer binding was not detected.

Because fragment $E 4$ was the region from intron 1 with strongest enhancer activity, we concentrated our analysis on those sites that were present in this core region and bound by Sox10. These were site 3 , site 4 , and site 7 . Mutations were introduced into the CAA core of each of these sites, and the consequence of these mutations on binding was then tested in EMSA. As evident from Figure $5 C$, all mutations effectively abrogated Sox 10 binding to the respective sites.

Mutations were introduced in the context of E4 into each of these sites as single mutations or in combinations, and the con- sequences on enhancer activity were assessed in luciferase reporter gene assays in transfected S16 cells (Fig. 6A). Compared with wild type, all E4 mutants exhibited dramatically reduced enhancer activity. Wild-type E4 enhanced the activity of the Dhh minimal promoter 150 -fold, but activation rates were down to sixfold for the site 3 mutant (Fig. 6A, E4m3). The site 4 mutation $(E 4 m 4)$ reduced activation rates to ninefold, and the site $7 \mathrm{mu}-$ tation $(E 4 m 7)$ allowed a residual 14-fold activation. E4 mutants with simultaneous mutation of two sites exhibited even lower activation rates. These results argue that $E 4$ enhancer activity in S16 cells depends on the three identified Sox10 binding sites.

We also analyzed to what extent the three mutations altered E4 responsiveness toward Sox 10 by cotransfecting all E4 mutants with the dominant-negative Sox 10 (Fig. 6 B). Sox 10 MIC not only effectively reduces the activity of wild-type $E 4$ but also the remaining activity of E4m4, E4m7, and the E4m4m7 double mutant. In contrast, the residual activity of the $E 4 \mathrm{~m} 3$ mutant or all $E 4$ double and triple mutants with mutant site 3 were refractory to Sox10 MIC. 

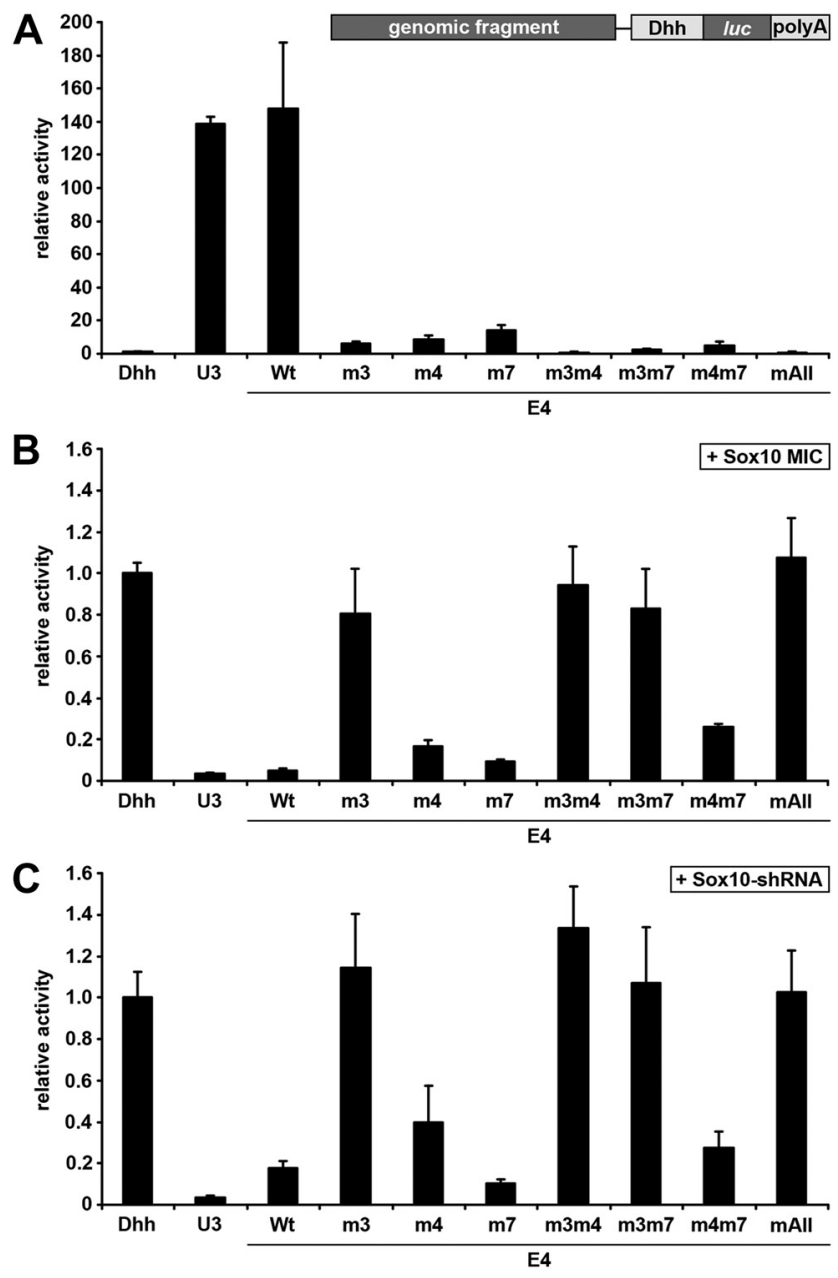

Figure 6. Functional relevance of Sox 10 binding sites in the Dhh enhancer. $\boldsymbol{A}$, Luciferase reporter constructs containing the $E 4$ fragment in wild-type or mutant versions were transiently transfected in $\mathrm{S} 16$ cells, and luciferase activities were determined $48 \mathrm{~h}$ after transfection. Mutant $E 4$ versions carried inactivating mutations in Sox 10 binding site $3(\mathrm{~m} 3)$, site 4 (m4), site 7 (m7), or combinations thereof ( $\mathrm{m} 3 \mathrm{~m} 4, \mathrm{~m} 3 \mathrm{~m} 7, \mathrm{~m} 4 \mathrm{~m} 7, \mathrm{mAll})$. The luciferase activity obtained for a reporter plasmid containing only the minimal Dhh promoter (Dhh) was arbitrarily set to 1. Activities in the presence of $E 4$ were calculated relative to minimal promoter activity and are presented as mean $\pm S D$. $\boldsymbol{B}$, The same luciferase reporter constructs were also transfected in $\mathrm{S} 16$ cells in the presence of a Sox $10 \mathrm{MIC}$ expression plasmid or an empty expression plasmid. The luciferase activity obtained for a reporter plasmid in the presence of empty expression plasmid was arbitrarily set to 1 . Activities in the presence of the Sox 10 MIC expression plasmid were normalized for each luciferase reporter to this value and are presented as mean \pm SD. $C$, Additionally, transfections of luciferase reporters were performed in the presence of Sox10specific shRNA and scrambled shRNA. Luciferase activities were determined, and the ratio of activities in the presence of Sox10-specific shRNA versus scrambled shRNA was calculated. Values were then normalized to the relative activity of the reporter plasmid containing only the minimal promoter and are presented as mean \pm SD. All experiments were performed three times in duplicates.

Very similar results were also obtained when S16 cells were transfected with the $E 4$ reporter plasmids in the presence of Sox10-specific shRNAs (Fig. 6C). The activity of the Dhh minimal promoter was only marginally affected by the presence of Sox10-shRNA or scrambled shRNA. For reporter plasmids that additionally contained the $U 3$ or the $E 4$ enhancer, activity was reduced to a residual 5-20\% in the presence of Sox10-shRNA. Similar to the wild-type, several E4 mutants were responsive to the Sox10-shRNA, arguing that they still contain residual functional Sox 10 binding sites. These included $E 4$ versions with site 4, site 7 , and combined site $4 / 7$ mutations. In contrast, site 3 muta- tions abolished this responsiveness when present alone or in combination with other mutations (Fig. 6C). Both cotransfections with Sox10-specific shRNA and Sox10 MIC therefore lead us to conclude that site 3 is most important among the three Sox10 binding sites present in E4.

Considering that we detected Sox10 binding to three sites and found mutation of these sites to reduce activity and Sox10 responsiveness, it is reasonable to assume that Sox 10 exerts its stimulatory effect on $E 4$ by direct binding. To verify this assumption, we performed ChIP from $\mathrm{S} 16$ cells with Sox 10-specific antibodies (Fig. $7 A, B$ ). These antibodies readily precipitated chromatin fragments from the $E 4$ enhancer, leading to their enrichment in the precipitate relative to two control fragments from the upstream region of the Dhh gene (Fig. $7 B$ ). No such enrichment was observed with control antibodies. Interestingly, the enrichment was much higher for the $E 4$ region that contained site 3 and site 4 than for the region with site 7 (Fig. $7 B$, compare E4a, E4b). ChIP thus confirms the presence of Sox10 on the E4 enhancer in S16 Schwann cells.

\section{Schwann-cell-specific activity of the Dhh enhancer depends on Sox10 in vivo}

Although the ChIP results in S16 cells are suggestive, they do not prove that E4 is truly active as a Sox10-dependent Schwann cell enhancer in vivo. To confirm enhancer activity in vivo, we generated transgenic constructs in which $E 4$ was placed in front of the Dhh minimal promoter and a lac $Z$ reporter gene (Fig. 7C, $E 4$-lacZ). Injection of the E4-lacZ transgene into the male pronucleus of fertilized oocytes allowed us to generate five transgenic embryos (Fig. $7 F$ ) that were analyzed at embryonic day 14.5 by $\mathrm{X}$-gal staining and immunohistochemistry (Fig. $7 D, E$ ). At the time of analysis, the embryos contained on average one to nine copies of the transgene per cell as determined by quantitative PCR (Fig. 7F). Four of the five embryos exhibited staining along the peripheral nerve. There was some variation in staining intensity and in the number of stained cells. Nevertheless, staining along the nerve was clearly detectable. Immunohistochemistry confirmed that $\beta$-galactosidase was present in the cytoplasm of those cells along the nerve that contain Sox10 in their nuclei and are thereby defined as Schwann cells (Fig. 7E). We therefore conclude that E4 is able to target transgene expression to Schwann cells in vivo.

In addition to transgenes with wild-type $E 4$, we also injected transgenes in which all three Sox10 binding sites (E4mAll-lacZ) or site 3 (E4m3-lacZ) were mutated (Fig. 7C). Of the four transgenic embryos obtained for E4mAll-lac $Z$, none exhibited significant activity in peripheral nerves (Fig. $7 D, F$ ). Similarly, none of the seven embryos obtained for E4m3-lac Z at embryonic day 14.5 exhibited expression of the lac $Z$ reporter along the nerve (Fig. $7 D, F)$. The activity observed for the lac $Z$ transgene in vivo thus crucially depends on $E 4$ and not the minimal promoter, and within $E 4$ on the identified Sox10 binding sites, in particular on site 3.

Finally, we electroporated the E4-lacZ transgene into the neural tube of early chicken embryos in the presence of GFP as electroporation marker. Considering that the neural tube at this stage consists of highly proliferative, Sox10-negative neuroepithelial cells, the lack of activity of the lac $Z$ transgene was not surprising (Fig. $7 G, H$ ). However, lacZ activity became readily visible in the electroporated half of the neural tube after coelectroporation of Sox10 (Fig. 7I,J), arguing that Dhh enhancer activity indeed depends on this transcription factor in vivo. 
A

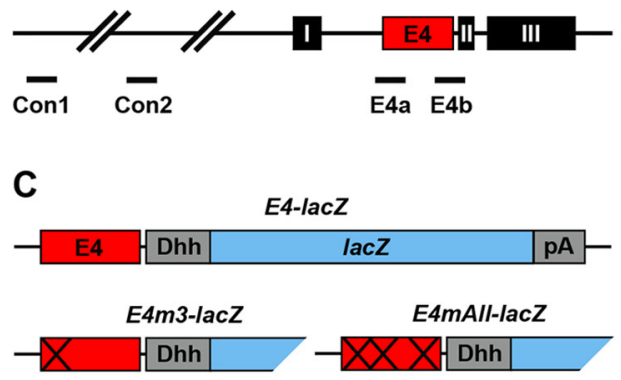

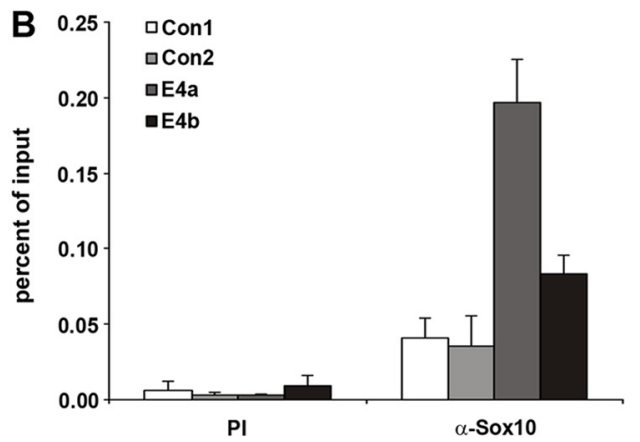

D
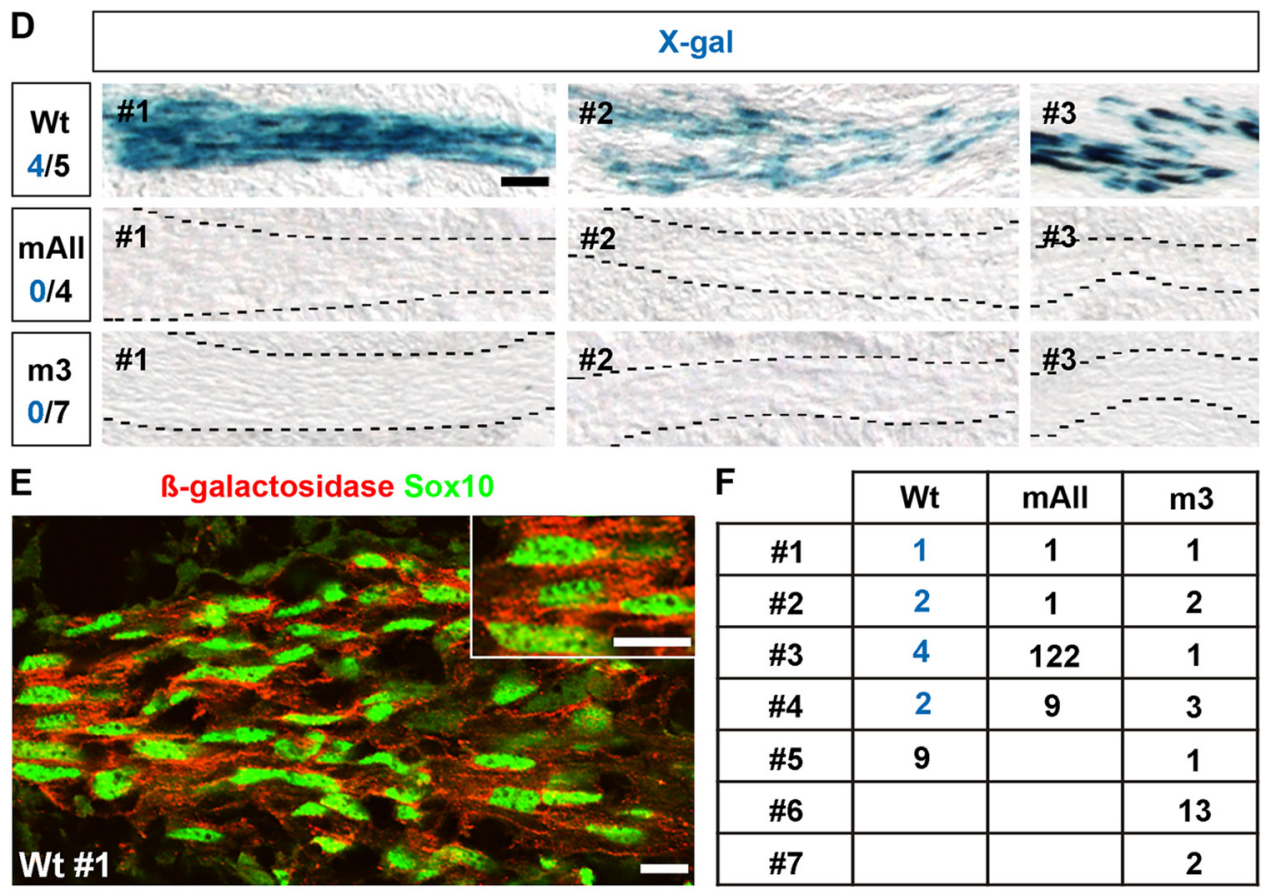

\begin{tabular}{|c|c|c|c|}
\hline & $\mathbf{W t}$ & mAll & $\mathrm{m} 3$ \\
\hline \#1 & 1 & 1 & 1 \\
\hline \#2 & 2 & 1 & 2 \\
\hline \#3 & 4 & 122 & 1 \\
\hline$\# 4$ & 2 & 9 & 3 \\
\hline \#5 & 9 & & 1 \\
\hline \#6 & & & 13 \\
\hline \#7 & & & 2 \\
\hline
\end{tabular}
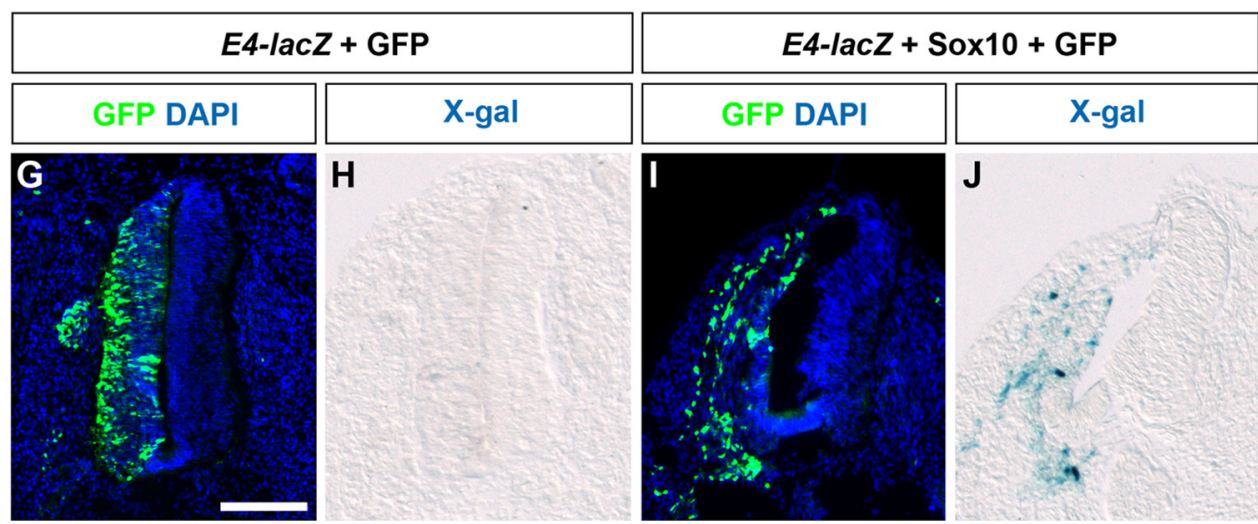

Figure 7. In vivo requirement of Sox 10 binding for Dhh activity. $A$, Schematic representation of the location of regions from the Dhh locus probed by PCR during ChIP studies, including the front (E4a, positions +2363 to +2779$)$ and hind (E4b, positions +2929 to +3293$)$ part of $E 4$ and two control fragments from the distal upstream region of the Dhh gene (Con1, positions -118661 to -119032; Con2, positions - 78544 to - 78886). B, ChIP was performed on S16 cells using antibodies directed against Sox10 ( $\alpha$-Sox10) and control preimmune serum (PI). Quantitative PCR was applied on the immunoprecipitate. Values for each fragment correspond to the percentage of material precipitated from the input. C, Schematic representation of the transgenic constructs consisting of $E 4$ in wild-type (E4) or mutant (E4m3 and E4mAll) versions, the minimal Dhh promoter (Dhh), the lacZ marker gene (lacZ), and an SV40 poly(A) signal (pA). D-F, Analysis of transgenic mouse embryos. D, LacZ activity was detected colorimetrically using X-gal substrate on transverse sections of 14.5-d-old transgenic mouse embryos. Only spinal nerve (marked by dotted lines) is shown. The number of embryos that exhibited X-gal staining in the nerve relative to the total number of transgenic embryos obtained for E4-lacZ (Wt), E4mAll-lacZ (mAll), and E4m3-lacZ (m3) by pronucleus injection are given on the left. Scale bar, $50 \mu \mathrm{m}$. $E$, Coimmunohistochemistry was performed on transverse sections of 14.5-d-old E4-lacZ mouse embryos using antibodies directed against $\beta$-galactosidase (in red) in combination with antibodies directed against Sox10 (in green). The confocal image is confined to spinal nerve. The inlay shows a higher resolution. Scale bars, 10 $\mu \mathrm{m} . \boldsymbol{F}$, Summary of mouse embryos obtained for each transgene and analyzed during this study including the determined number of transgene copies present on average in each embryo per cell. G-J, Analysis of electroporated chicken embryos. Chicken embryos were electroporated with the E4-lacZ in the presence of $p C A G G S-I R E S-n / s-G F P(G, H)$ or $p C A G G S-S o x 10-I R E S-n / s-G F P(I, J)$. Electroporated neural tube regions were identified $48 \mathrm{~h}$ later on transverse sections by unilateral GFP expression (green) using DAPl as a nuclear counterstain (blue) $(\mathbf{G}, I)$. LacZ activity was detected on immediately adjacent sections using X-gal substrate $(\boldsymbol{H}, \boldsymbol{J})$. Scale bar (in $\mathbf{G})$ : $\mathbf{G}-\mathbf{J}, 200 \mu \mathrm{m}$. 


\section{Discussion}

In this study, we have mapped the regulatory region that is likely responsible for Dhh expression in Schwann cells by a combination of cell culture and transgenic studies. Schwann-cell-specific expression was not mediated by the promoter but rather by an enhancer within the first intron of the Dhh gene. The enhancer was localized in the hind part of intron 1, and the $E 4$ region constituted its core.

It is unclear at present whether $E 4$ is identical to the enhancer or whether surrounding sequences additionally contribute to enhancer activity. Two observations argue for a role of E4 flanking sequences in enhancer activity. Although E4 effectively directed reporter gene expression to Schwann cells in transgenic embryos, expression levels were quite variable. This is a consequence of different copy numbers and integration sites in the transgenic embryos. However, the fact that activity is fairly sensitive to these parameters may indicate that E4 lacks some of the elements that stabilize enhancer activity in situ. Additionally, E4 is embedded in a larger region that exhibits sequence conservation among mammalian species, and this conservation may reflect the exact dimensions of the enhancer.

It is also noteworthy that conservation was only observed among mammals. Already in monotherians, the conserved region is much smaller than in metatherians and confined to $190 \mathrm{bp}$ of $E 4$ surrounding site 3 and site 4 . No conservation was detected in other vertebrates in agreement with the fact that Dhh is a hedgehog gene specific to mammals (O'Hara et al., 2011).

We have also shown that the Dhh enhancer and its E4 core contain binding sites for Sox 10 and that they respond to its presence. As a consequence, deletion of the Sox10 binding sites led to a dramatic decrease of enhancer activity in cultured cells and abolished Schwann-cell-specific activity in vivo. Considering further that Sox10 was detected on this enhancer by ChIP and that the presence of Sox10 allows this enhancer to function in neuroepithelial cells after electroporation in vivo, there is strong evidence that Sox10 contributes to the Schwann-cell-specific activity of the Dhh enhancer by directly binding to these sites.

Among the three sites identified, site 3 is the most important for Sox10-dependent activity, despite a rather modest Sox10 binding in vitro when compared with site 4 and site 7 . This shows that binding strength in vitro is not a completely reliable predictor for functional importance in vivo. Several reasons for the discrepancy can be envisaged. It is possible that the accessibility of the sites is different in chromatin in vivo compared with naked DNA in vitro. Some of the sites may be more effectively masked in nucleosomes than others. Binding of other transcription factors to the enhancer has to be taken into account as well. Competitive binding to overlapping sites may reduce accessibility, whereas transcription factor binding to adjacent sites may allow additional contacts between the two bound proteins and thereby facilitate binding. In this respect, it is intriguing that there is a potential binding site for Krox20 adjacent to site 3 . Both sites are separated by $11 \mathrm{bp}$, and the distance between their centers corresponds exactly to two helical turns. This is closer than most previously identified composite binding elements for Sox10 and Krox20 (Jones et al., 2007). It also differs from previously identified sites in that it combines the Krox20 site with a monomeric rather than a dimeric Sox10 binding site. Additional sites for Klf, Nfat, and YY1 proteins are a bit farther away. Future experiments will have to probe their relevance.

It is also worth mentioning that all of the identified sites bind Sox 10 as monomer. Similar to the other SoxE proteins Sox 8 and
Sox9, Sox10 can bind to DNA as monomer or dimer (Wegner, 2010), and it has been shown for at least two Sox10-responsive enhancers that a dimer site cannot simply be replaced by a monomer site without a significant loss in enhancer activity (Peirano and Wegner, 2000; Jagalur et al., 2011). It has even been postulated that dimeric binding is such a crucial feature that it can be used to predict Sox10-dependent neural-crest enhancers (Antonellis et al., 2008). For Schwann-cell-specific enhancers that are activated by Sox 10, there seems to be no strict reliance on dimeric binding. Some Schwann-cell-specific enhancers only contain dimer sites and crucially depend on them (i.e., the Oct6 SCE) (Jagalur et al., 2011), whereas others contain only monomeric sites (i.e., the Dhh enhancer), yet others carry both monomeric and dimeric sites and need both for their activity (i.e., Krox20 myelinating Schwann cell enhancer, U3 enhancer of the Sox10 gene) (Reiprich et al., 2010; Wahlbuhl et al., 2012). Considering that Sox 10 monomers affect the overall topology of the enhancer in a different way than dimers, the most likely explanation is that each enhancer contains the type of site that is best suited at its specific position to guarantee the multiprotein complex formation of the enhanceosome.

The identification of Dhh as a direct target gene also provides the first mechanistic explanation for a non-cell-autonomus function of Sox10. By regulating Dhh expression in Schwann cells, Sox10 influences the development of perineurial cells and is responsible for formation and proper function of the perineurium. Loss or inactivation of Sox10 should therefore be associated invariably with defects in the perineurial sheath. The compromised blood-nerve and tissue-nerve barriers should furthermore lead to elevated immune cell numbers in the nerve, which in turn may evoke a chronic inflammation. The fact that these changes are indeed observed in mouse models with Sox10-deficient Schwann cells (Finzsch et al., 2010) confirms the relevance of this effectortarget gene relationship for normal nerve development. Disturbance of Dhh signaling to the perineurial cells may also occur in patients with Sox10 mutations and contribute to disease manifestation and pathology in humans.

\section{References}

Antonellis A, Huynh JL, Lee-Lin SQ, Vinton RM, Renaud G, Loftus SK, Elliot G, Wolfsberg TG, Green ED, McCallion AS, Pavan WJ (2008) Identification of neural crest and glial enhancers at the mouse Sox10 locus through transgenesis in zebrafish. PLoS Genet 4:e1000174.

Bermingham JR Jr, Scherer SS, O’Connell S, Arroyo E, Kalla KA, Powell FL, Rosenfeld MG (1996) Tst-1/Oct-6/SCIP regulates a unique step in peripheral myelination and is required for normal respiration. Genes Dev 10:1751-1762.

Bitgood MJ, McMahon AP (1995) Hedgehog and Bmp genes are coexpressed at many diverse sites of cell-cell interaction in the mouse embryo. Dev Biol 172:126-138.

Bondurand N, Girard M, Pingault V, Lemort N, Dubourg O, Goossens M (2001) Human Connexin 32, a gap junction protein altered in the $\mathrm{X}$-linked form of Charcot-Marie-Tooth disease, is directly regulated by the transcription factor SOX10. Hum Mol Genet 10:2783-2795.

Bremer M, Fröb F, Kichko T, Reeh P, Tamm ER, Suter U, Wegner M (2011) Sox10 is required for Schwann cell homeostasis and myelin maintenance in the adult peripheral nerve. Glia 59:1022-1032.

Britsch S, Goerich DE, Riethmacher D, Peirano RI, Rossner M, Nave KA, Birchmeier C, Wegner M (2001) The transcription factor Sox10 is a key regulator of peripheral glial development. Genes Dev 15:66-78.

Carney TJ, Dutton KA, Greenhill E, Delfino-Machín M, Dufourcq P, Blader P, Kelsh RN (2006) A direct role for Sox10 in specification of neural crest-derived sensory neurons. Development 133:4619-4630.

Cossais F, Wahlbuhl M, Kriesch J, Wegner M (2010) SOX10 structurefunction analysis in the chicken neural tube reveals important insights into its role in human neurocristopathies. Hum Mol Genet 19:2409-2420. 
Finzsch M, Stolt CC, Lommes P, Wegner M (2008) Sox9 and Sox10 influence survival and migration of oligodendrocyte precursors in the spinal cord by regulating PDGF receptor $\alpha$ expression. Development 135:637-646.

Finzsch M, Schreiner S, Kichko T, Reeh P, Tamm ER, Bösl MR, Meijer D, Wegner M (2010) Sox10 is required for Schwann cell identity and progression beyond the immature Schwann cell stage. J Cell Biol 189:701-712.

Fröb F, Bremer M, Finzsch M, Kichko T, Reeh P, Tamm ER, Charnay P, Wegner M (2012) Establishment of myelinating Schwann cells and barrier integrity between central and peripheral nervous systems depend on Sox10. Glia 60:806-819.

Ghislain J, Charnay P (2006) Control of myelination in Schwann cells: a Krox 20 cis-regulatory element integrates Oct6, Brn2 and Sox 10 activities. EMBO Rep 7:52-58.

Inoue K, Khajavi M, Ohyama T, Hirabayashi S, Wilson J, Reggin JD, Mancias P, Butler IJ, Wilkinson MF, Wegner M, Lupski JR (2004) Molecular mechanism for distinct neurological phenotypes conveyed by allelic truncating mutations. Nat Genet 36:361-369.

Jaegle M, Mandemakers W, Broos L, Zwart R, Karis A, Visser P, Grosveld F, Meijer D (1996) The POU factor Oct-6 and Schwann cell differentiation. Science 273:507-510.

Jaegle M, Ghazvini M, Mandemakers W, Piirsoo M, Driegen S, Levavasseur F, Raghoenath S, Grosveld F, Meijer D (2003) The POU proteins Brn-2 and Oct-6 share important functions in Schwann cell development. Genes Dev 17:1380-1391.

Jagalur NB, Ghazvini M, Mandemakers W, Driegen S, Maas A, Jones EA, Jaegle M, Grosveld F, Svaren J, Meijer D (2011) Functional dissection of the Oct6 Schwann cell enhancer reveals an essential role for dimeric Sox 10 binding. J Neurosci 31:8585-8594.

Jones EA, Jang SW, Mager GM, Chang LW, Srinivasan R, Gokey NG, Ward RM, Nagarajan R, Svaren J (2007) Interactions of Sox10 and Egr2 in myelin gene regulation. Neuron Glia Biol 3:377-387.

Jones EA, Lopez-Anido C, Srinivasan R, Krueger C, Chang LW, Nagarajan R, Svaren J (2011) Regulation of the PMP22 gene through an intronic enhancer. J Neurosci 31:4242-4250.

Kim J, Lo L, Dormand E, Anderson DJ (2003) SOX10 maintains multipotency and inhibits neuronal differentiation of neural crest stem cells. Neuron 38:17-31.

Kuhlbrodt K, Herbarth B, Sock E, Hermans-Borgmeyer I, Wegner M (1998a) Sox10, a novel transcriptional modulator in glial cells. J Neurosci $18: 237-250$

Kuhlbrodt K, Schmidt C, Sock E, Pingault V, Bondurand N, Goossens M, Wegner M (1998b) Functional analysis of Sox10 mutations found in human Waardenburg-Hirschsprung patients. J Biol Chem 273: 23033-23038.

Küspert M, Hammer A, Bösl MR, Wegner M (2011) Olig2 regulates Sox10 expression in oligodendrocyte precursors through an evolutionary conserved distal enhancer. Nucleic Acids Res 39:1280-1293.

LeBlanc SE, Ward RM, Svaren J (2007) Neuropathy-associated Egr2 mutants disrupt cooperative activation of myelin protein zero by Egr2 and Sox10. Mol Cell Biol 27:3521-3529.

Maka M, Stolt CC, Wegner M (2005) Identification of Sox8 as a modifier gene in a mouse model of Hirschsprung disease reveals underlying molecular defect. Dev Biol 277:155-169.

Newbern J, Birchmeier C (2010) Nrg1/ErbB signaling networks in Schwann cell development and myelination. Semin Cell Dev Biol 21:922-928.

O'Hara WA, Azar WJ, Behringer RR, Renfree MB, Pask AJ (2011) Desert hedgehog is a mammal-specific gene expressed during testicular and ovarian development in a marsupial. BMC Dev Biol 11:72.
Paratore C, Goerich DE, Suter U, Wegner M, Sommer L (2001) Survival and glial fate acquisition of neural crest cells are regulated by an interplay between the transcription factor Sox 10 and extrinsic combinatorial signaling. Development 128:3949-3961.

Parmantier E, Lynn B, Lawson D, Turmaine M, Namini SS, Chakrabarti L, McMahon AP, Jessen KR, Mirsky R (1999) Schwann cell-derived Desert hedgehog controls the development of peripheral nerve sheaths. Neuron 23:713-724.

Peirano RI, Wegner M (2000) The glial transcription factor Sox10 binds to DNA both as monomer and dimer with different functional consequences. Nucleic Acids Res 28:3047-3055.

Peirano RI, Goerich DE, Riethmacher D, Wegner M (2000) Protein zero expression is regulated by the glial transcription factor Sox10. Mol Cell Biol 20:3198-3209.

Prasad MK, Reed X, Gorkin DU, Cronin JC, McAdow AR, Chain K, Hodonsky CJ, Jones EA, Svaren J, Antonellis A, Johnson SL, Loftus SK, Pavan WJ, McCallion AS (2011) SOX10 directly modulates ERBB3 transcription via an intronic neural crest enhancer. BMC Dev Biol 11:40.

Reiprich S, Stolt CC, Schreiner S, Parlato R, Wegner M (2008) SoxE proteins are differentially required in mouse adrenal gland development. Mol Biol Cell 19:1575-1586.

Reiprich S, Kriesch J, Schreiner S, Wegner M (2010) Activation of Krox20 gene expression by Sox10 in myelinating Schwann cells. J Neurochem 112:744-754

Schlierf B, Ludwig A, Klenovsek K, Wegner M (2002) Cooperative binding of Sox10 to DNA: requirements and consequences. Nucleic Acids Res 30:5509-5516.

Schlierf B, Werner T, Glaser G, Wegner M (2006) Expression of Connexin 47 in oligodendrocytes is regulated by the Sox 10 transcription factor. J Mol Biol 361:11-21.

Schreiner S, Cossais F, Fischer K, Scholz S, Bösl MR, Holtmann B, Sendtner M, Wegner M (2007) Hypomorphic Sox10 alleles reveal novel protein functions and unravel developmental differences in glial lineages. Development 134:3271-3281.

Sharghi-Namini S, Turmaine M, Meier C, Sahni V, Umehara F, Jessen KR, Mirsky R (2006) The structural and functional integrity of peripheral nerves depends on the glial-derived signal desert hedgehog. J Neurosci 26:6364-6376.

Stolt CC, Wegner M (2010) SoxE function in vertebrate nervous system development. Int J Biochem Cell Biol 42:437-440.

Stolt CC, Rehberg S, Ader M, Lommes P, Riethmacher D, Schachner M, Bartsch U, Wegner M (2002) Terminal differentiation of myelinforming oligodendrocytes depends on the transcription factor Sox10. Genes Dev 16:165-170.

Svaren J, Meijer D (2008) The molecular machinery of myelin gene transcription in Schwann cells. Glia 56:1541-1551.

Umehara F, Tate G, Itoh K, Yamaguchi N, Douchi T, Mitsuya T, Osame M (2000) A novel mutation of desert hedgehog in a patient with 46,XY partial gonadal dysgenesis accompanied by minifascicular neuropathy. Am J Hum Genet 67:1302-1305.

Wahlbuhl M, Reiprich S, Vogl MR, Bösl MR, Wegner M (2012) Transcription factor Sox 10 orchestrates activity of a neural crest-specific enhancer in the vicinity of its gene. Nucleic Acids Res 40:88-101.

Wegner M (2010) All purpose Sox: the many roles of Sox proteins in gene expression. Int J Biochem Cell Biol 42:381-390.

Werner T, Hammer A, Wahlbuhl M, Bösl MR, Wegner M (2007) Multiple conserved regulatory elements with overlapping functions determine Sox10 expression in mouse embryogenesis. Nucleic Acids Res 35:65266538 . 